\title{
Association between chemotherapy and cognitive impairments in a large cohort of patients with colorectal cancer
}

\author{
XIANGLIN L. DU, YI CAI and ELAINE SYMANSKI \\ School of Public Health, Division of Epidemiology, Human Genetics and Environmental Sciences, \\ University of Texas Health Science Center, Houston, TX, USA
}

Received December 20, 2012; Accepted February 8, 2013

DOI: $10.3892 /$ ijo.2013.1882

\begin{abstract}
No population-based study has been conducted on the relationship between chemotherapy and the risk of cognitive impairments in patients with colorectal cancer. This study aimed to determine this association in a large populationbased cohort of patients. We studied 72,374 men and women who were diagnosed with stages I-III colorectal cancer at age $\geq 65$ years from 1991 through 2002 from 16 regions in the Surveillance, Epidemiology and End Results program who were free of cognitive impairments at baseline with up to 17 years of follow-up and also studied 15,921 matched cohorts based on the propensity of receiving chemotherapy. The cumulative incidence of drug-induced dementia at 5 years was 16.2 cases per 1,000 persons for the chemotherapy group and 12.4 cases per 1,000 persons for the no chemotherapy group. Overall, patients who received chemotherapy were $24 \%$ significantly more likely to develop drug-induced dementia compared to those without chemotherapy after adjusting for patient and tumor characteristics (hazard ratio 1.24,95\% confidence interval 1.05-1.47). The significantly increased risk was only observed in those without mood disorder who received chemotherapy in the entire cohort $(1.26,1.06-1.50)$ and in the matched cohort $(1.29,1.04-1.59)$. The risk of developing Alzheimer's disease, vascular dementia or other dementias was significantly lower in patients receiving chemotherapy compared to those without chemotherapy regardless of mood disorder status. In conclusion, there was a significant association between chemotherapy and the risk of developing drug-induced dementia in patients with colorectal cancer without mood disorder, but chemotherapy was associated with a decreased risk of other dementias.
\end{abstract}

Correspondence to: Professor Xianglin L. Du, School of Public Health, Division of Epidemiology, Human Genetics and Environmental Sciences, University of Texas Health Science Center, 1200 Herman Pressler Drive, RAS-E631, Houston, TX 77030, USA

E-mail: xianglin.l.du@uth.tmc.edu

Key words: chemotherapy, dementia, cognitive impairments, elderly people, colorectal cancer

\section{Introduction}

Chemotherapy has been documented to be efficacious in treating men and women with stage III colon cancer (1-5) and the clinical guidelines are clear about recommending adjuvant chemotherapy for these patients after resection $(1,5)$. For patients with stages II-III rectal cancer, the combinedmodality of adjuvant chemotherapy and radiation therapy is currently the standard of care $(1,5)$. Although chemotherapy is not well documented in randomized trials for those with stage I or II colon cancer (6-11), a substantial number of patients with stage II colon cancer received chemotherapy $(12,13)$. What has not been well studied is the potential association between chemotherapy utilization and the risk of cognitive impairments in patients with cancer treated with chemotherapy. There have been some anecdotal reports since the 1980s, indicating that patients treated with chemotherapy complained of changes in their memory, attention, concentration, and language skills primarily in those with cancer (14-23). The first study that examined this relationship within the context of a randomized trial was reported by van Dam and colleagues in 1998 for patients with breast cancer (24). In the treatment group, they administered four cycles of combination chemotherapy agents (fluorouracil, doxorubicin and cyclophosphamide); and a fifth course of high-dose combination chemotherapy (cyclophosphamide and carboplatin) (24). The subsequent small-scale studies seemed to support the original findings (25-32). However, minor cognitive impairments (such as minor decrease in memory, attention, concentration and language skills) may be reversible after a short time use of chemotherapy, even if there were true causal relationships. No clinical trials have been conducted to determine the association between chemotherapy and long-term follow-up of outcomes such as dementia, which are unlikely to be reversed.

There have been four population-based studies examining a long-term relationship between chemotherapy and dementia (33-36) and one of these studies examined the 5 types of cognitive impairments (36), all of which were conducted in breast cancer patients. No nationwide large population-based study has been conducted on this research question in patients with colorectal cancer. Because similar chemotherapy agents such as fluorouracil are commonly used in patients with colorectal cancer as well as in patients with breast cancer, it is reasonable to posit that, if chemotherapy use increased the risk 
of cognitive impairments in patients with breast cancer, this relationship will likely be observed in those with colorectal cancer who received similar chemotherapy agents. Therefore, this study aimed to primarily determine the long-term risk of drug-induced dementia associated with chemotherapy use and also explore if the use of chemotherapy was associated with the risks of other cognitive impairments, including Alzheimer's disease, in a large population-based cohort of patients with colorectal cancer in the US with up to 17 years of follow-up. In this study, only those patients who were free of any cognitive impairment at baseline were included. Moreover, additional matched-cohort analysis according to the propensity of receiving chemotherapy was conducted to verify the study findings and to assess the potential impact of selection bias that might have affected the study findings. Our hypothesis was that there were significant differences in the occurrence of developing cognitive impairments in men and women diagnosed with colorectal cancer who received chemotherapy compared to those who did not use chemotherapy.

\section{Patients and methods}

Data sources and study population. The National Cancer Institute's Surveillance, Epidemiology and End Results (SEER) 16 cancer registries and Medicare linked databases were used for this analysis $(37,38)$. The Committee for the Protection of Human Subjects at the University of Texas Health Science Center at Houston approved this study.

The study population consisted of 120,111 patients who were diagnosed with colorectal cancer as the only primary tumor at age $\geq 65$ years from 1991 through 2002. Cases from Atlanta and rural Georgia were combined due to small numbers in the same state. We excluded 35,475 subjects who did not have full coverage of Medicare Parts A and B or were enrolled with Health Maintenance Organizations from the year of diagnosis to the last follow-up (December 2006 or date of death). For the purpose of this study in determining the relationship between chemotherapy and cognitive impairments, we included only patients who were free of any cognitive impairment at the time of cancer diagnosis. By doing so, we excluded 4,078 cases with preexisting cognitive impairments, 5,129 patients aged $\geq 90$ and 3,073 cases who received first chemotherapy after 12 months of diagnosis, leaving 72,374 subjects for the final analysis.

Matched cohort. In order to minimize selection bias due to factors that may have influenced physicians or patients to choose chemotherapy, we first calculated the propensity (or conditional probability) of receiving chemotherapy for all patients, and then matched patients who actually received chemotherapy with those who had the same or similar propensity but did not receive chemotherapy. The propensity of receiving chemotherapy was created through the logistic regression model based on the following patient and tumor characteristics: age, sex, ethnicity, marital status, tumor stage, tumor grade, tumor size, number of positive lymph nodes, comorbidity, surgery, radiotherapy, socioeconomic status, cancer type, year of diagnosis and SEER areas. The matching through the 5-1 digit propensity of receiving chemotherapy was performed using the greedy matching algorithm by
Parsons (39). A total of 15,921 patients receiving chemotherapy were matched with 15,921 patients who did not receive chemotherapy.

Chemotherapy. The methods of identifying chemotherapy use through the Medicare claims was discussed elsewhere (40) and the validity of Medicare claims for chemotherapy have been reasonably well confirmed (41-48).

Cognitive impairments and mood disorders. Cognitive impairment was defined if there were at least two claims in all Medicare claim files (including inpatient, outpatient and physician claims) that were 30 days apart for each of the following diagnoses (with ICD-9-CM codes) (46) after chemotherapy use: unspecified cognitive disorder (294.9), amnestic disorder (294.0), Alzheimer's disease (331.0), vascular dementia (290.x), unspecified dementia (294.8) or drug-induced dementia and psychoses (292.x).

Mood disorder was defined if there were at least two claims that were 30 days apart for each of the following diagnoses (46) before or after chemotherapy use: anxiety state, unspecified (300.0); anxiety depression (300.4); unspecified depressive disorder, (311.x); alteration of consciousness (780.9); and other depressions (296.2, 296.3, 296.5-296.7, 298.0, 301.10, 301.12, 301.13, 309.0, 309.1).

Other variables. Comorbidity was ascertained from Medicare claims data through diagnoses or procedures that were made between 1 year prior to and 1 month after the diagnosis of colorectal cancer using the previously validated comorbidity index (49-51) and SAS macro program (52). The percent of persons living below the poverty line at the census tract level from the 1990 census for cases in 1991-1999 and from the 2000 census for cases in 2000-2002 was used to define the socioeconomic status (SES). These percentages were then classified into quartiles.

Analyses. The $\chi^{2}$ statistic (at a significance level of 0.05) was used to compare baseline characteristics between patients who received chemotherapy and those who did not in the entire cohort and in the matched cohort. Incidence rate (density) was defined as the ratio of the number of new cognitive impairments over the total number of person-years. Person-years were calculated as the number of patients multiplied by the number of years from diagnosis to the date of the first cognitive impairment or date of death or date of last follow-up, whichever occurred first. The cumulative incidence (probability) of cognitive impairments was calculated using the statistical program by Penman and Johnson (53). The time to event (cognitive impairment) analysis was conducted using the Cox proportional hazard regression model available in SAS (54).

\section{Results}

Table I presents the distribution of baseline characteristics among the entire cohort of patients with colorectal cancer according to chemotherapy status and also presents the comparisons of the matched cohort based on propensity score of receiving chemotherapy. In the entire cohort, a significantly 
Table I. Comparisons of characteristics among women with colon cancer according to the receipt of chemotherapy (chemo) in both entire cohort and propensity-matched cohort.

\begin{tabular}{|c|c|c|c|c|c|c|}
\hline \multirow[b]{2}{*}{ Characteristics } & \multicolumn{3}{|c|}{ Column $\%$ of the entire cohort } & \multicolumn{3}{|c|}{ Column $\%$ of the matched cohort } \\
\hline & $\begin{array}{c}\text { Chemo } \\
(n=23,484)\end{array}$ & $\begin{array}{l}\text { No chemo } \\
(\mathrm{n}=48,890)\end{array}$ & P-value & $\begin{array}{c}\text { Chemo } \\
(n=15,921)\end{array}$ & $\begin{array}{l}\text { No chemo } \\
(n=15,921)\end{array}$ & P-value \\
\hline Median age (range) & $73(65-89)$ & $78(65-89)$ & & $75(65-89)$ & $75(65-89)$ & \\
\hline \multicolumn{7}{|l|}{ Age (years) } \\
\hline $65-69$ & 28.1 & 14.4 & $<0.001$ & 21.4 & 21.4 & 0.817 \\
\hline $70-74$ & 30.7 & 19.8 & & 27.5 & 27.4 & \\
\hline $75-79$ & 24.7 & 24.2 & & 27.9 & 27.8 & \\
\hline $80-84$ & 12.8 & 24.1 & & 17.7 & 17.6 & \\
\hline $85-89$ & 3.7 & 17.4 & & 5.4 & 5.8 & \\
\hline \multicolumn{7}{|l|}{ Gender } \\
\hline Male & 48.6 & 43.9 & $<0.001$ & 47.2 & 47.1 & 0.893 \\
\hline Female & 51.4 & 56.1 & & 52.8 & 52.9 & \\
\hline \multicolumn{7}{|l|}{ Race/ethnicity } \\
\hline Caucasians & 85.1 & 84.3 & $<0.001$ & 84.4 & 84.1 & 0.725 \\
\hline African Americans & 6.7 & 8.1 & & 7.5 & 7.8 & \\
\hline Others & 8.2 & 7.6 & & 8.1 & 8.1 & \\
\hline \multicolumn{7}{|l|}{ Marital status } \\
\hline Married & 59.3 & 45.2 & $<0.001$ & 53.7 & 53.3 & 0.712 \\
\hline Unmarried & 37.9 & 50.5 & & 42.9 & 43.1 & \\
\hline Unknown & 2.8 & 4.3 & & 3.4 & 3.5 & \\
\hline \multicolumn{7}{|c|}{ Socioeconomic status (SES) } \\
\hline First quartile (high) & 26.8 & 23.6 & $<0.001$ & 24.7 & 24.5 & 0.789 \\
\hline Second quartile & 25.2 & 24.3 & & 24.8 & 24.6 & \\
\hline Third quartile & 24 & 24.9 & & 24.9 & 24.8 & \\
\hline Fourth quartile (low) & 22.5 & 25.5 & & 24.1 & 24.5 & \\
\hline Missing SES & 1.4 & 1.7 & & 1.5 & 1.6 & \\
\hline \multicolumn{7}{|l|}{ Tumor stage } \\
\hline I & 5.5 & 33.0 & $<0.001$ & 8.2 & 7.8 & 0.676 \\
\hline II & 22.9 & 29.4 & & 29.7 & 29.8 & \\
\hline III & 41.0 & 12.9 & & 29.2 & 28.9 & \\
\hline IV & 25.7 & 15.8 & & 26.7 & 27.1 & \\
\hline Unstaged & 4.9 & 8.8 & & 6.2 & 6.4 & \\
\hline \multicolumn{7}{|l|}{ Tumor size $(\mathrm{cm})$} \\
\hline$<1$ & 0.5 & 2.9 & $<0.001$ & 0.7 & 0.7 & 0.959 \\
\hline $1-<2$ & 2.1 & 4.1 & & 2.3 & 2.2 & \\
\hline $2-<3$ & 7.6 & 8.6 & & 7.5 & 7.4 & \\
\hline $3-<4$ & 14.6 & 12.9 & & 13.7 & 13.8 & \\
\hline$\geq 4$ & 56.1 & 41.0 & & 53.7 & 53.9 & \\
\hline Missing & 19.0 & 30.6 & & 22.1 & 22.0 & \\
\hline \multicolumn{7}{|c|}{$\mathrm{N}$ of positive lymph nodes } \\
\hline 0 (negative) & 27.1 & 50.6 & $<0.001$ & 35.6 & 35.1 & 0.889 \\
\hline 1 & 14.9 & 5.4 & & 12.1 & 12 & \\
\hline $2-3$ & 16.7 & 5.4 & & 12.2 & 12.1 & \\
\hline $4-9$ & 15.8 & 5.0 & & 11.4 & 11.5 & \\
\hline $10-51$ & 6.7 & 2.6 & & 5.5 & 5.7 & \\
\hline Missing & 18.8 & 31.1 & & 23.2 & 23.5 & \\
\hline
\end{tabular}


Table I. Continued.

\begin{tabular}{|c|c|c|c|c|c|c|}
\hline \multirow[b]{2}{*}{ Characteristics } & \multicolumn{3}{|c|}{ Column $\%$ of the entire cohort } & \multicolumn{3}{|c|}{ Column $\%$ of the matched cohort } \\
\hline & $\begin{array}{c}\text { Chemo } \\
(n=23,484)\end{array}$ & $\begin{array}{l}\text { No chemo } \\
(n=48,890)\end{array}$ & P-value & $\begin{array}{c}\text { Chemo } \\
(n=15,921)\end{array}$ & $\begin{array}{l}\text { No chemo } \\
(n=15,921)\end{array}$ & P-value \\
\hline
\end{tabular}

Tumor grade

Well-differentiated

Moderately-differentiated

Poorly-differentiated

Unknown/missing

Comorbidity scores

0

1

$\geq 2$

Primary surgery (resection)

No

Yes

$$
5.5
$$

61.5

24.6

8.4

61.5

25.4

12.1

11.3

88.7

71.7

28.3

7.2

7.3

6.9

6.6

7.0

6.6

6.8

7.0

6.0

10.2

14.0

14.4

2002

\section{SEER areas}

\section{Connecticut}

Detroit

Hawaii

Iowa

New Mexico

Seattle

Utah

Atlanta/rural Georgia

Kentucky

Louisiana

New Jersey

California

Cancer type

Colon cancer

Rectal cancer

Total

12.2

14.0

2.1

13.0

2.9

7.4

3.1

4.7

3.6

2.8

7.5

26.7

69.9

30.1

100.0

5

$\begin{aligned} 9.9 & <0.001 \\ 56.4 & \end{aligned}$

6.2

60.1

22.8

10.8

$<0.001$

57.6

26.3

16.1

26.7

22.3

17.6

$<0.001$

14.5

85.5

82.4

88.6

$<0.001$

11.4

79.6

20.4

7.4

7.2

6.6

6.6

6.7

6.6

6.8

6.5

6.1

12.0

13.5

13.8

13.3

11.8

12.8

2.0

13.6

3.4

7.5

3.5

4.4

3.8

2.9

7.1

27.2

77.1

22.9

100.0
11.5

13.6

2.0

12.9

3.2

7.2

3.3

4.6

3.8

2.8

7.4

27.7

$<0.001$

74.6

25.4

100.0
15.0

85.0

79.5

20.5

$$
\begin{array}{r}
6.0 \\
60.1 \\
23.0 \\
10.9
\end{array}
$$

0.815

57.2

26.6

16.2

(5)

85.0

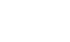

7.6

7.4

6.9

6.6

6.8

6.2

6.8

6.6

5.9

11.9

13.8

13.5

12.1

13.2

1.9

12.7

3.2

7

3.2

4.8

3.9

2.9

7.2

27.8

74.3

25.7

100.0 

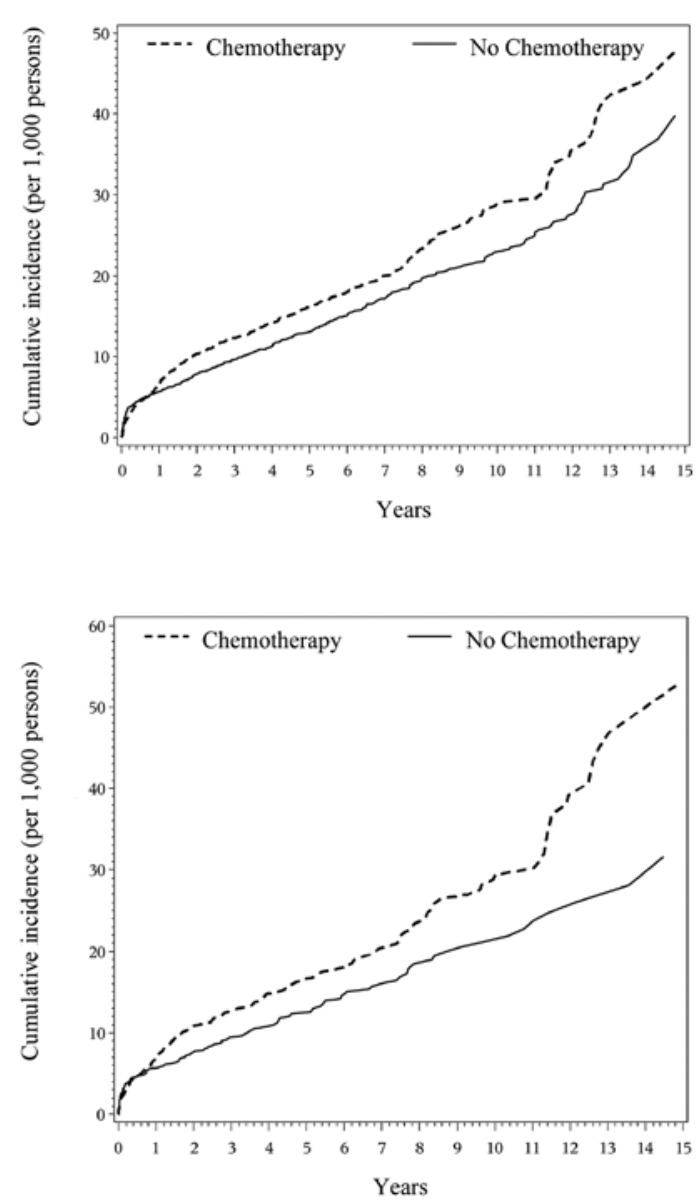

Figure 1. Cumulative incidence of drug-induced dementia in patients with colorectal cancer, by chemotherapy status. Top, entire cohort; bottom, matched cohort.

higher proportion of younger patients and married people received chemotherapy. A slightly higher percentage of women and Caucasians received chemotherapy. The higher percentage of not receiving chemotherapy was related to lower socioeconomic status, earlier tumor stage, smaller tumor size, fewer positive lymph nodes, low grade tumors and higher comorbidity scores. A higher proportion of patients who had resection and radiation therapy also received chemotherapy. The distribution of these characteristics was significantly different between patients receiving chemotherapy and those who did not. However, in the matched cohort, there were no significant differences between these two groups in terms of all baseline characteristics.

Table II presents the incidence density of cognitive impairments by chemotherapy status, age, gender, tumor stage and comorbidity. The incidence density of drug-induced dementia was higher in patients receiving chemotherapy than those who did not receive chemotherapy in all strata of age, sex, stage and comorbidity score. For example, the incidence of druginduced dementia in patients aged 65-69 was 1.84 times higher in patients receiving chemotherapy than in those not receiving chemotherapy (3.33 versus 1.81 per 10,000 person-years), whereas the relative risk was 1.42 in patients aged $80-84$ years (5.15 versus 3.63 per 10,000 person-years) between the two groups. However, the incidence of other types of cognitive impairments such as Alzheimer's disease, vascular disorder,
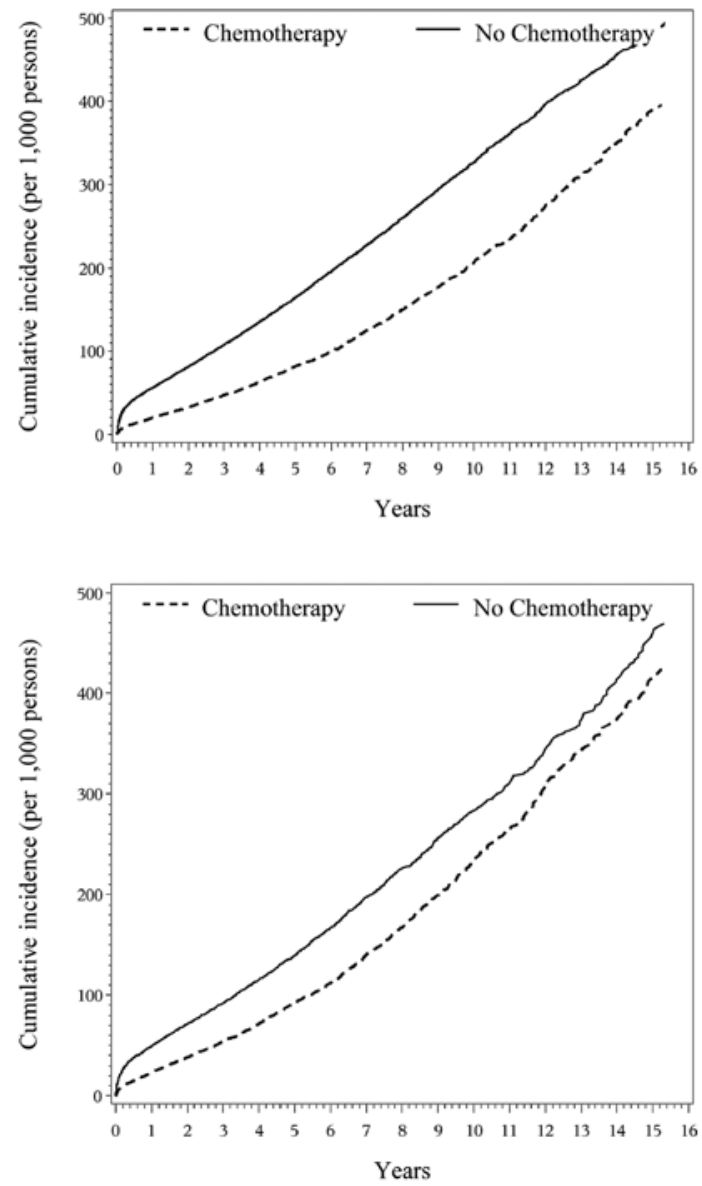

Figure 2. Cumulative incidence of any dementia in patients with colorectal cancer, by chemotherapy status. Top, entire cohort; bottom, matched cohort.

cognitive disorder or other dementia appeared to be higher in patients who did not receive chemotherapy. Overall, the incidence rate of various cognitive impairments increased with advanced age and higher comorbidity scores but was relatively similar across gender and tumor stage.

Fig. 1 presents the cumulative incidence curve of druginduced dementia over the 15 -year period by chemotherapy status. The probability of drug-induced dementia was similar for chemotherapy group compared to no chemotherapy group in the first year. After the first year, the incidence of druginduced dementia became higher in the chemotherapy group than in the no chemotherapy group and the gap between the two groups appeared to widen over time (Fig. 1, top). The cumulative incidence of drug-induced dementia at 3 years was 12.3 cases per 1,000 persons for the chemotherapy group and 9.7 cases per 1,000 persons for no chemotherapy group, while the cumulative incidence was 16.2 and 13.0 per 1,000 persons at 5 years and 29.0 and 22.8 per 1,000 persons at 10 years for both groups. Similar curves were observed among the matched cohorts (Fig. 1, bottom). However, the patterns of cumulative incidence were different for four other types of cognitive impairments, in which the incidence was lower in patients receiving chemotherapy than those without chemotherapy except for cognitive disorder in which incidence probability overlapped in years 7-12 in both the entire and matched cohorts (data not shown). Fig. 2 presents the cumulative incidence 
Table II. Incidence density of cognitive impairments by chemotherapy status and other factors in the entire cohort of patients with colon cancer.

Incidence density of cognitive impairments

(number of cases per 1,000 person-years), by chemotherapy status

\begin{tabular}{lccccc}
\cline { 2 - 5 } $\begin{array}{l}\text { Patient and tumor } \\
\text { characteristics }\end{array}$ & $\begin{array}{c}\text { Drug-induced } \\
\text { dementia }\end{array}$ & $\begin{array}{c}\text { Alzheimer's } \\
\text { disease }\end{array}$ & $\begin{array}{c}\text { Vascular } \\
\text { dementia }\end{array}$ & $\begin{array}{c}\text { Cognitive } \\
\text { disorder, NOS }\end{array}$ & $\begin{array}{c}\text { Other dementias } \\
\text { or dementia, NOS }\end{array} \quad \begin{array}{c}\text { Any dementia } \\
(\text { any of these 5) }\end{array}$ \\
\hline
\end{tabular}

Chemotherapy

Age (years)

65-69 3.33

$70-74$

3.33

$75-79$

2.87

3.24

6.09

3.92

0.84

0.71

4.76

11.16

10.06

0.49

8.06

16.89

80-84

5.15

12.58

13.75

26.89

85-89

3.91

15.02

19.25

1.44

20.67

41.83

0.43

26.44

59.63

Gender

Male

3.52

Female

3.45

Tumor stage

I

II

2.70

2.80

III

3.16

IV

7.82

Unstaged

3.42

Comorbidity scores

0

1

$\geq 2$

Total

No chemotherapy

Age (years)

$\begin{array}{ll}65-69 & 1.81 \\ 70-74 & 2.65 \\ 75-79 & 2.70 \\ 80-84 & 3.63 \\ \geq 85 & 3.77\end{array}$

Gender

Male

Female

2.76

2.86

8.33

0.81

0.73

11.47

0.56

1.07

0.57

0.71

1.21

5.89

11.4

9.12

6.68

8.44

9.45

7.49

11.04

15.43

7.38

9.14

0.7

1.04

0.59

0.77

Tumor stage

$\begin{array}{ll}\text { I } & 2.40 \\ \text { II } & 2.88 \\ \text { III } & 3.74 \\ \text { IV } & 8.65 \\ \text { Unstaged } & 2.12\end{array}$

Comorbidity scores

\begin{tabular}{cc}
0 & 2.22 \\
1 & 3.19 \\
$\geq 2$ & 4.73 \\
Total & 2.82 \\
\hline
\end{tabular}

${ }^{a}$ NOS, not otherwise specified.

2.40

2.88

8.65

2.12

2.22

3.19

2.82
12.08

15.46

4.13

8.90

14.98

22.43

25.84

$\begin{array}{rrrrr}4.13 & 6.43 & 0.71 & 5.95 & 12.82 \\ 8.90 & 12.74 & 0.82 & 12.29 & 24.37 \\ 14.98 & 22.22 & 1.02 & 20.49 & 40.59 \\ 22.43 & 35.01 & 1.57 & 31.87 & 63.85 \\ 25.84 & 48.15 & 1.41 & 43.44 & 89.29 \\ & & & & \\ 12.08 & 18.86 & 1.09 & 17.61 & 35.84 \\ 15.46 & 24.43 & 1.05 & 22.39 & 44.08 \\ & & & & \\ 11.67 & 16.91 & 0.89 & 16.21 & 31.17 \\ 14.75 & 23.62 & 1.01 & 21.66 & 42.59 \\ 20.45 & 33.01 & 1.81 & 31.11 & 60.89 \\ 18.72 & 38.06 & 0.96 & 31.58 & 84.29 \\ 18.13 & 35.3 & 1.88 & 27.28 & 64.14 \\ & & & & \\ 12.30 & 16.8 & 0.85 & 16.28 & 31.53 \\ 16.14 & 27.03 & 1.22 & 22.96 & 47.51 \\ 17.74 & 36.15 & 1.70 & 33.30 & 68.01 \\ 14.03 & 22.06 & 1.07 & 20.36 & 40.56\end{array}$

24.37

40.59

63.85

89.29

35.84

4.08

14.03
20.09

21.64

22.1

20.54

20.56

20.5

26.81

17.74

23.98

34.55

20.91

10.10

40.56 
curve of any dementia (all above dementias combined) over the 15 -year period by chemotherapy status. The results were similar to those from the four types of cognitive impairments other than drug-induced dementia.

Table III presents the time-to-event analysis for the hazard ratio of having various types of cognitive impairments. Patients who received chemotherapy were $24 \%$ significantly more likely to develop drug-induced dementia compared to those without chemotherapy after adjusting for patient and tumor characteristics (hazard ratio 1.24, 95\% CI 1.05-1.47). On the contrast, the risk of developing Alzheimer's disease, vascular dementia, or other dementias was significantly lower in patients receiving chemotherapy than that in those who did not receive chemotherapy, except for cognitive disorder which was not significantly different between the two groups. As expected, the risk of all types of cognitive impairments increased significantly with age and comorbidity scores. There were no significant differences in the risk of developing drug-induced dementia, Alzheimer's disease and vascular dementia between men and women, but women appeared to have slightly lower risks of cognitive disorders and other non-specified dementias. The risk of all types of cognitive impairments was not significantly associated with the receipt of radiation therapy.

Table IV presents the risks of developing cognitive impairments in patients who were treated with chemotherapy compared to those who were not, stratified by the status of mood disorder. The risk of developing drug-induced dementia seemed to be only significantly elevated in those without mood disorder who received chemotherapy compared to those who did not receive chemotherapy in both the entire cohort (hazard ratio 1.26, 95\% CI 1.06-1.50) and the matched cohort (1.29, 1.04-1.59). In those who had a history of mood disorder, there was no longer a significant difference in the risk of developing drug-induced dementia between the chemotherapy and no chemotherapy groups $(1.13,0.58-2.18$ for the entire cohort; $1.29,0.52-3.20$ for the matched cohort). On the other hand, the risk of developing Alzheimer's disease, vascular dementia or other unspecified dementias was significantly lower in patients receiving chemotherapy compared to those without chemotherapy while the risk of cognitive disorder was not significantly different between the two groups. Also, there was no evidence of effect modification by status of mood disorder. The findings from any dementia (all above dementias combined) were similar to those from the four types of cognitive impairments other than drug-induced dementia.

\section{Discussion}

This study found that patients who received chemotherapy were $24 \%$ significantly more likely to develop drug-induced dementia compared to those without chemotherapy after adjusting for patient and tumor characteristics. The significantly increased risk was limited to those without a history of mood disorder. The risk of developing Alzheimer's disease, vascular dementia or other dementias was significantly lower in patients receiving chemotherapy than those who did not receive chemotherapy, except for cognitive disorder that was not significantly different between the two groups, which all were not affected by the history of mood disorder. These findings should have important clinical and public health implications, including key messages about potential chemotherapy-induced dementia but no evidence about chemotherapy associated with the increased risk of other dementias.

Possible relationships between cognitive impairments and chemotherapy have been examined in clinical or community settings involving patients treated with chemotherapy for breast cancer (24-36) and none was conducted in men or women with colorectal cancer. It is important to note that previous pioneering small-scale clinical trials in the 1990s showed a significant association between chemotherapy use and cognitive impairments (24-31). Those small trials with short follow-up times mostly tested the changes in memory and attention which could be reversed after stopping chemotherapy. Our study followed patients up to 17 years after cancer diagnosis, therefore making it possible to examine long-term cognitive impairments such as dementia at the late clinical stages.

The link between chemotherapy and cognitive impairments and its potential mechanisms have been examined in animal models (55-60). For example, it was suggested that deficits in DNA-repair mechanisms and/or a deregulated immune response, coupled with the effect of chemotherapy on these systems, might have contributed to cognitive decline in rats following chemotherapy (56). In another study (59), cyclophosphamide- and doxorubicin-treated rats showed significantly impaired performance on the novel place recognition task compared with untreated controls, suggesting a significant decline in neurogenesis in chemotherapy-treated animals. However, a study by Fremouw et al (57) showed that despite significant toxic effects, chemotherapy-treated mice performed as well as control mice on all tasks, concluding that as are some humans, these mice may be resistant to at least some aspects of chemotherapy-induced cognitive decline. Moreover, the study by Fardell et al (58) showed that exercising rats had improved cognition relative to non-exercising rats after fluorouracil and oxaliplatin, suggesting that physical activity may help ameliorate the cognitive impairments induced by chemotherapy.

Our study has a number of strengths. First, the study population covered a large cohort of community-based Medicare beneficiaries, leading to much greater generalization of the study findings to the elderly population aged $\geq 65$ across the country. Second, a unique feature of our study was that we included only those patients free of any cognitive impairment at the time of cancer diagnosis, thus leading to clearer causeeffect temporal relationships. Third, because of potential selection bias in which some patients with certain characteristics were given chemotherapy while other patients were not given this therapy according to preferences by patients or providers, or because of potential confounding by indication in which whether or not chemotherapy was given was influenced by other conditions such as cognitive decline, mood disorder or other comorbid conditions, the matched cohort analyses based on the conditional probability of receiving chemotherapy would minimize selection bias and potential confounding. In addition, the analysis was further stratified by the status of mood disorder which demonstrated important differences in the associations between chemotherapy use and cognitive impairments. Finally, a large population-based cohort of patients with colorectal cancer were followed-up from 4 to 17 years, allowing for more time to capture chronic 


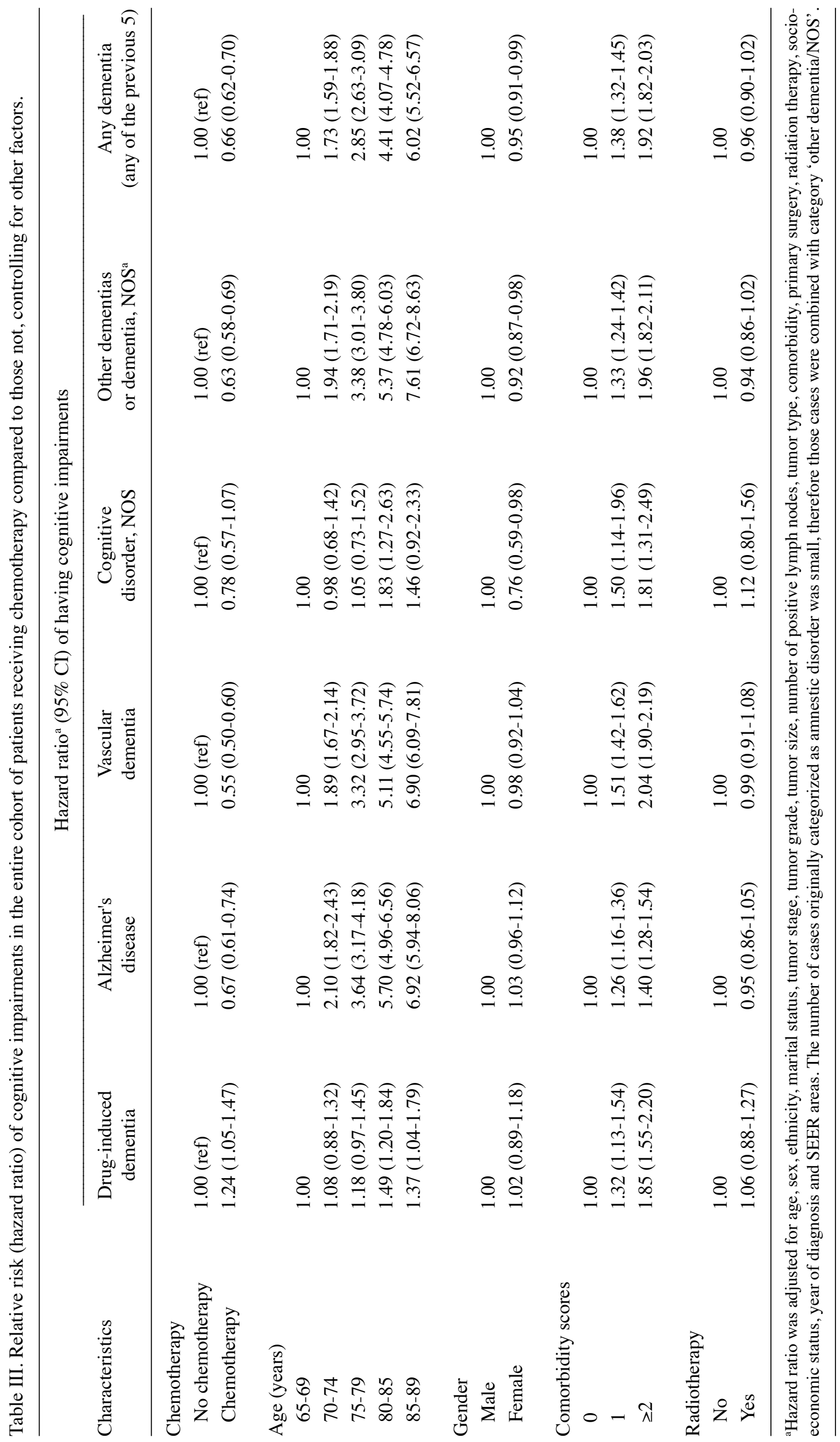


Table IV. Hazard ratio of developing cognitive impairments in entire cohort and matched cohort of patients with colon cancer who received chemotherapy compared to those without receiving chemotherapy, stratified by the status of mood disorder.

Hazard ratio $(95 \% \mathrm{CI})^{\mathrm{a}}$ of having cognitive impairments in patients receiving chemotherapy compared to those who did not,

by status of mood disorder prior to cancer diagnosis

Type of cognitive impairments

$\begin{array}{cc}\text { Had mood disorder } & \text { No mood disorder } \\ (\mathrm{n}=2,908) & (\mathrm{n}=69,466) \\ 1.13(0.58-2.18) & 1.26(1.06-1.50) \\ 0.66(0.44-1.00) & 0.68(0.61-0.75) \\ 0.49(0.35-0.68) & 0.56(0.51-0.61) \\ 0.91(0.22-3.74) & 0.78(0.56-1.09) \\ 0.66(0.48-0.91) & 0.64(0.59-0.69) \\ 0.61(0.48-0.77) & 0.67(0.63-0.71) \\ (\mathrm{n}=1,193) & (\mathrm{n}=30,649) \\ 1.29(0.52-3.20) & 1.29(1.04-1.59) \\ 0.58(0.35-0.98) & 0.65(0.58-0.73) \\ 0.44(0.29-0.67) & 0.53(0.48-0.58) \\ - & 0.77(0.53-1.13) \\ 0.60(0.41-0.90) & 0.61(0.55-0.67) \\ 0.51(0.41-0.74) & 0.64(0.60-0.69)\end{array}$

Entire cohort

Drug-induced dementia

Alzheimer's disease

Vascular dementia

Cognitive disorder- NOS

Other dementias or dementia NOS

Any dementia (any of above)

Matched cohort

Drug-induced dementia

Alzheimer's disease

Vascular dementia

Cognitive disorder, NOS

Other dementias or dementia NOS

Any dementia (any of above)

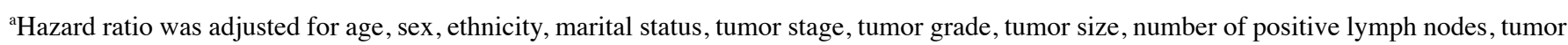
type, comorbidity, primary surgery, radiation therapy, socioeconomic status, year of diagnosis and SEER areas.

conditions such as dementia that would be otherwise missed in short follow-up studies.

It is important to note several important limitations of this study. First, study outcomes only included more serious and late-stage cognitive impairments (such as Alzheimer's disease), which might not be comparable to early stage of cognitive impairments identified in previous clinical trials $(19-21,25,26)$. The claims data did not allow assessment of the early-stage disease process (such as decline in memory or attention) of cognitive impairments. Due to this limitation, our study population who were free of dementia at the baseline might have included those who already had early stage cognitive impairments, which could have affected the association between chemotherapy and outcomes. Second, even though we adjusted for some measured confounding factors and used the matched cohort analysis based on the probability of receiving chemotherapy, there could have been unmeasured or unknown factors which could have influenced physicians to prescribe chemotherapy or not to do so. In particular, if physicians were aware of some studies on potential link between chemotherapy and cognitive impairments, they might hesitate to prescribe chemotherapy to those with suspected cognitive problems. Hence, the potential for selection bias could not be ruled out in this study. Third, the finding that the risk of drug-induced dementia was significantly increased only in those patients without mood disorder may be vulnerable to surveillance bias because of the link between mood disorder and dementia (61). In other words, patients without a history of mood disorder could be more likely labeled by the treating clinicians or coders as having 'drug-induced dementia', whereas those with a history of mood disorder might be more likely labeled as having dementias other than drug-induced. Similarly, because of difficulty in confirming if a dementia was induced by chemotherapy, clinical or coding staff might likely code a dementia as drug-induced for patients receiving chemotherapy but code differently for patients without receiving chemotherapy, leading to certain degrees of differential misclassification of study outcomes. One way to see if this was the case is to analyze all dementias combined regardless of drug-induced one. We performed additional analyses and found that the receipt of chemotherapy was associated with the decreased risk of all cognitive impairments combined (Tables II-IV and Fig. 2). Fourth, Medicare claims had limited information on the dosage and intensity of chemotherapy that could have affected the occurrence and severity of cognitive impairments. We relied on the common procedure codes that specified the standard dose for each chemotherapy agent but in practice the treating physician might have modified the chemotherapy doses according to each patient's characteristics. Furthermore, this study did not examine the types of chemotherapy utilized, the number of cycles administered, and their potential effects on the outcomes.

In conclusion, there was a significant association between chemotherapy and the risk of developing drug-induced dementia in patients with colorectal cancer who received chemotherapy but did not have a history of mood disorder. This study with long-term follow-up found that the risks of Alzheimer's disease, vascular dementia, or other non-specified 
dementias were even lower in patients with colorectal cancer with chemotherapy use than those without this therapy.

\section{Acknowledgements}

We acknowledge the efforts of the National Cancer Institute; Center for Medicare and Medicaid Services; Information Management Services, Inc.; and the SEER Program tumor registries in the creation of this database. The interpretation and reporting of these data are the sole responsibilities of the authors. This study was supported in part by a grant from the Agency for Healthcare Research and Quality (R01-HS018956) and in part by a grant from the Cancer Prevention and Research Institute of Texas (RP101207).

\section{References}

1. NIH Consensus Conference: Adjuvant therapy for patients with colon and rectal cancer. JAMA 264: 1444-1450, 1990.

2. Moertel CG, Fleming TR, Macdonald JS, et al: Fluorouracil plus levamisole as effective adjuvant therapy after resection of stage III colon carcinoma: a final report. Ann Intern Med 122: 321-326, 1995.

3. Sargent DJ, Goldberg RM, Jacobson SD, et al: A pooled analysis of adjuvant chemotherapy for resected colon cancer in elderly patients. N Engl J Med 345: 1091-1097, 2001.

4. Gill S, Loprinzi CL, Sargent DJ, et al: Pooled analysis of fluorouracil-based adjuvant therapy for stage II and III colon cancer: who benefits and by how much? J Clin Oncol 22: 1797-1806, 2004.

5. National Cancer Institute: Colon and Rectal cancer Treatment. http://www.cancer.gov/cancertopics/treatment/colon-and-rectal. Accessed September 16, 2012.

6. O'Connell MJ, Martenson JA, Wieand HS, et al: Improving adjuvant therapy for rectal cancer by combining protracted-infusion fluorouracil with radiation therapy after curative surgery. N Engl J Med 331: 502-507, 1994.

7. Figueredo A, Charette ML, Maroun J, Brouwers MC and Zuraw L: Adjuvant therapy for stage II colon cancer: a systematic review from the Cancer Care Ontario Program in evidence-based care's gastrointestinal cancer disease site group. J Clin Oncol 22: 3395-3407, 2004.

8. Benson AB III, Schrag D, Somerfield MR, et al: American Society of Clinical Oncology recommendations on adjuvant chemotherapy for stage II colon cancer. J Clin Oncol 22: 3408-3419, 2004.

9. Sargent DJ, Wieand HS, Haller DG, et al: Disease-free survival versus overall survival as a primary end point for adjuvant colon cancer studies: individual patient data from 20,898 patients on 18 randomized trials. J Clin Oncol 23: 8664-8670, 2005.

10. Goldberg RM, Tabah-Fisch I, Bleiberg H, et al: Pooled analysis of safety and efficacy of oxaliplatin plus fluorouracil/leucovorin administered bimonthly in elderly patients with colorectal cancer. J Clin Oncol 24: 4085-4091, 2006.

11. André T, Boni C, Navarro M, et al: Improved overall survival with oxaliplatin, fluorouracil, and leucovorin as adjuvant treatment in stage II or III colon cancer in the MOSAIC trial. J Clin Oncol 27: 3109-3116, 2009.

12. Schrag D, Rifas-Shiman S, Saltz L, Bach PB and Begg CB: Adjuvant chemotherapy use for Medicare beneficiaries with stage II colon cancer. J Clin Oncol 20: 3999-4005, 2002.

13. O'Connor ES, Greenblatt DY, LoConte NK, et al: Adjuvant chemotherapy for stage II colon cancer with poor prognostic features. J Clin Oncol 29: 3381-3388, 2011.

14. Silberfarb PM, Philibert D and Levine PM: Psychosocial aspects of neoplastic disease: II. Affective and cognitive effects of chemotherapy in cancer patients. Am J Psychiatry 137: 597-601, 1980.

15. Oxman TE and Silberfarb PM: Serial cognitive testing in cancer patients receiving chemotherapy. Am J Psychiatry 137: 1263-1265, 1980.

16. Devlen J, Maguire P, Phillips P and Crowther D: Psychological problems associated with diagnosis and treatment of lymphomas. II: Prospective study. Br Med J 295: 955-957, 1987.
17. Parth P, Dunlap WP, Kennedy RS, et al: Motor and cognitive testing of bone marrow transplant patients after chemoradiotherapy. Percept Mot Skills 68: 1227-1241, 1989.

18. Copeland DR, Moore BD III, Francis DJ, et al: Neuropsy-chologic effects of chemotherapy on children with cancer: a longitudinal study. J Clin Oncol 14: 2826-2835, 1996.

19. Waber DP, Tarbell NJ, Fairclough D, et al: Cognitive sequelae of treatment in childhood acute lymphoblastic leukemia: cranial radiation requires an accomplice. J Clin Oncol 13: 2490-2496, 1995.

20. Ahles TA, Silberfarb PM, Rundle AC, et al: Quality of life in patients with limited small-cell carcinoma of the lung receiving chemotherapy with or without radiation therapy, for cancer and Leukemia Group B. Psychother Psychosom 62: 193-199, 1994.

21. Wieneke MH and Dienst ER: Neuropsychological assessment of cognitive functioning following chemotherapy for breast cancer. Psychooncology 4: 61-66, 1995.

22. Meyers CA, Byrne KS and Komaki R: Cognitive deficits in patients with small cell lung cancer before and after chemotherapy. Lung Cancer 12: 231-235, 1995.

23. Schwartz CL: Late effects of treatment in long-term survivors of cancer. Cancer Treat Rev 21: 355-366, 1995.

24. van Dam FS, Schagen SB, Muller MJ, et al: Impairment of cognitive function in women receiving adjuvant treatment for high-risk breast cancer: high-dose versus standard-dose chemotherapy. J Natl Cancer Inst 90: 210-218, 1998.

25. Schagen SB, van Dam FS, Muller MJ, et al: Cognitive deficits after postoperative adjuvant chemotherapy for breast carcinoma. Cancer 85: 640-650, 1999.

26. Schagen SB, Hamburger HL, Muller MJ, et al: Neurophysiological evaluation of late effects of adjuvant high-dose chemotherapy on cognitive function. J Neurooncol 51: 159-165, 2001.

27. Schagen SB, Muller MJ, Boogerd W, et al: Change in cognitive function after chemotherapy: a prospective longitudinal study in breast cancer patients. J Natl Cancer Inst 98: 1742-1745, 2006.

28. Kreukels BP, van Dam FS, Ridderinkhof KR, et al: Persistent neurocognitive problems after adjuvant chemotherapy for breast cancer. Clin Breast Cancer 8: 80-87, 2008.

29. Brezden CB, Phillips KA, Abdolell M, et al: Cognitive function in breast cancer patients receiving adjuvant chemotherapy. J Clin Oncol 18: 2695-2701, 2000.

30. Wefel JS, Lenzi R, Theriault RL, Davis RN and Meyers CA: The cognitive sequelae of standard-dose adjuvant chemotherapy in women with breast carcinoma: results of a prospective, randomized, longitudinal trial. Cancer 100: 2292-2299, 2004.

31. Hurria A, Rosen C, Hudis C, et al: Cognitive function of older patients receiving adjuvant chemotherapy for breast cancer: a pilot prospective longitudinal study. J Am Geriatr Soc 54: 925-931, 2006.

32. Deprez S, Amant F, Smeets A, et al: Longitudinal assessment of chemotherapy-induced structural changes in cerebral white matter and its correlation with impaired cognitive functioning. J Clin Oncol 30: 274-281, 2012.

33. Heck JE, Albert SM, Franco R and Gorin SS: Patterns of dementia diagnosis in surveillance, epidemiology, and end results breast cancer survivors who use chemotherapy. J Am Geriatr Soc 56: 1687-1692, 2008.

34. Baxter NN, Durham SB, Phillips KA, Habermann EB and Virning BA: Risk of dementia in older breast cancer survivors: a population-based cohort study of the association with adjuvant chemotherapy. J Am Geriatr Soc 57: 403-411, 2009.

35. Raji MA, Tamborello LP, Kuo YF, Ju H, Freeman JL, Zhang DD, Giordano SH and Goodwin JS: Risk of subsequent dementia diagnoses does not vary by types of adjuvant chemotherapy in older women with breast cancer. Med Oncol 26: 452-459, 2009.

36. Du XL, Xia R and Hardy D: Relationship between chemotherapy use and cognitive impairments in older women with breast cancer: findings from a large population-based cohort. Am J Clin Oncol 33: 533-543, 2010.

37. National Cancer Institute: About the SEER (Surveillance Epidemiology and End Results) Registries. http://seer.cancer.gov/ registries/. Accessed September 15, 2012.

38. Warren JL, Klabunde CN, Schrag D, Bach PB and Riley GF: Overview of the SEER-Medicare data: content, research applications, and generalizability to the United States elderly population. Med Care 40 (Suppl): 3-18, 2002.

39. Parsons LS: Reducing Bias in a Propensity Score Matching-Pair Sample Using GreedyMatching Techniques, available at http:// www2.sas.com/proceedings/sugi26/p214-26.pdf. Accessed July 2, 2012. 
40. Du XL and Goodwin JS: Patterns of use of chemotherapy for breast cancer in older women: findings from Medicare claims data. J Clin Oncol 19: 1455-1461, 2001.

41. Warren JL, Harlan LC, Fahey A, et al: Utility of the SEERMedicare data to identify chemotherapy use. Med Care 40 IV-55-IV-61, 2002.

42. Du XL, Key CR, Dickie L, et al: External validation of medicare claims for breast cancer chemotherapy compared with medical chart reviews. Med Care 44: 124-131, 2006.

43. Lamont EB, Herndon JE II, Weeks JC, et al: Criterion validity of Medicare chemotherapy claims in cancer and leukemia Group B breast and lung cancer trial participants. J Natl Cancer Inst 97: 1080-1083, 2005.

44. Liang SY, Phillips KA, Wang G, et al: Tradeoffs of using administrative claims and medical records to identify the use of personalized medicine for patients with breast cancer. Med Care 49: e1-e8, 2011.

45. Lund JL, Stürmer T, Harlan LC, et al: Identifying specific chemotherapeutic agents in medicare data: a validation study. Med Care: Nov 10, 2011 (Epub ahead of print).

46. US Public Health Services: International Classification of Diseases, 9th Revision, Clinical Modification. 5th edition. Practice Management Information Corp., Los Angeles, CA, 1996.

47. American Medical Association: Physicians' Current Procedural Terminology-CPT 2000. American Medical Association, Chicago, IL, 2000.

48. Health Care Financing Administration: HCFA Common Procedure Coding System: National Level II Medicare Codes. Practice Management Information Corp., Los Angeles, CA, 2000.

49. Charlson ME, Pompei P, Ales KL and MacKenzie CR: A new method of classifying prognostic comorbidity in longitudinal studies: development and validation. J Chronic Dis 40: 373-383, 1987.

50. Romano PS, Roos LL and Jollis JG: Adapting a clinical comorbidity index for use with ICD-9-CM administrative data: differing perspectives. J Clin Epidemiol 46: 1075-1079, 1993.
51. Klabunde CN, Potosky AL, Legler JM and Warren JL: Development of a comorbidity index using physician claims data. J Clin Epidemiol 53: 1258-1267, 2000.

52. National Cancer Institute. SEER-Medicare: Calculation of Comorbidity Weights. http://healthservices.cancer.gov/seermedicare/program/comorbidity.html. Accessed July 18, 2012.

53. Penman AD and Johnson WD: A SAS program for calculating cumulative incidence of events (with confidence limits) and number at risk at specified time intervals with partially censored data. Comput Methods Programs Biomed 89: 50-55, 2008.

54. Allison PD: Survival Analysis Using the SAS System: A Practical Guide. SAS Institute Inc., Cary, NC, 1995.

55. Ahles TA and Saykin AJ: Candidate mechanisms for chemotherapy-induced cognitive changes. Nat Rev Cancer 7: 192-201, 2007.

56. Long JM, Lee GD, Kelley-Bell B, Spangler EL, Perez EJ, Longo DL, de Cabo R, Zou S and Rapp PR: Preserved learning and memory following 5-fluorouracil and cyclophosphamide treatment in rats. Pharmacol Biochem Behav 100: 205-211, 2011.

57. Fremouw T, Fessler CL, Ferguson RJ and Burguete Y: Preserved learning and memory in mice following chemotherapy: 5-fluorouracil and doxorubicin single agent treatment, doxorubicin-cyclophosphamide combination treatment. Behav Brain Res 226: 154-162, 2012.

58. Fardell JE, Vardy J, Shah JD and Johnston IN: Cognitive impairments caused by oxaliplatin and 5-fluorouracil chemotherapy are ameliorated by physical activity. Psychopharmacology 220 : 183-193, 2012.

59. Christie LA, Acharya MM, Parihar VK, Nguyen A, Martirosian V and Limoli CL: Impaired cognitive function and hippocampal neurogenesis following cancer chemotherapy. Clin Cancer Res 18: 1954-1965, 2012.

60. Winocur G, Henkelman M, Wojtowicz JM, Zhang H, Binns MA and Tannock IF: The effects of chemotherapy on cognitive function in a mouse model: a prospective study. Clin Cancer Res 18: 3112-3121, 2012

61. Byers AL and Yaffe K: Depression and risk of developing dementia. Nat Rev Neurol 7: 323-331, 2011. 